\title{
COMPLICAÇÕES DECORRENTES DO USO DE PRÓTESE VOCAL ${ }^{1}$.
}

\author{
COMPLICATIONS IN CONSEQUENCE OF THE VOCAL \\ PROSTHESIS USE.
}

\author{
Leonardo de Souza Kruschewsky ${ }^{2}$ \\ Luiz Carlos Conti de Freitas ${ }^{3}$ \\ Ernandes Nakamura ${ }^{3}$ \\ Rui Celso Martins Mamede ${ }^{4}$ \\ Francisco Veríssimo de Mello-Filho ${ }^{4}$ \\ Lílian Rics $^{5}$
}

\section{RESUMO}

Introdução - Desde a primeira laringectomia total, realizada em 1873, já se tem registro do interesse em se desenvolver e recuperar a comunicabilidade verbal desses pacientes. Porém grandes progressos foram observados depois de 1979, quando se pode contar com próteses traqueoesofágicas. Mesmo sendo um enorme progresso, as próteses vocais geram complicações. Objetivo - Avaliar as complicações com o emprego de próteses vocais e relaciona-las com fatores clínicos e tipo de prótese. Métodos - Dez pacientes submetidos a laringectomia total portando prótese fonatória foram acompanhados no serviço de Cirurgia de Cabeça e Pescoço do HCRP-FMRP-USP buscando-se registrar as complicações e relaciona-las com fatores clínicos e tipo de prótese. Resultados - Foram registrados: vazamento de saliva e/ou dieta da faringe para a traquéia, infecção fungica, ausência de função da prótese e esses achados foram quantificados avaliando-se os efeitos da radioterapia e do tipo de prótese usada. Conclusão - A prótese vocal apresenta dificuldades e complicações operacionais que merecem atenção e mais estudos são necessários para se ter o perfil mais completo destes aspectos. Disponível em URL: http://www.scielo.br/acb

Descritores - Laringe artificial; Laringectomia; Fonação

\begin{abstract}
Introduction - Since 1873 when the first total laryngectomy was performed there is a scientific interest in develop and recover patients' verbal comunication. Great successes were done after 1979, that permitted the tracheoesophagical prosthesis be a reality. Even being a special conquest the vocal prosthesis has its complications. Aim - To evaluate its complications registered with the vocal prosthesis use and its relation with patients' clinical aspects and the prosthesis kind. Methods - Ten total laryngectomised patients from the HCRP-FMRP-USP, Head and Neck ambulatory, were followed to complications manifestations with the prosthesis use. Results Complications as saliva and/or diet passage from the pharynx to the trachea, fungal infection, prosthesis dysfunction were registered and quantified in relation with radiotherapy and the prosthesis kind used. Conclusion - Even being a special development for patients' comunication and socialization, it offers some difficulties and complications that have to be observed and more studies are necessary to better evaluate these aspects.
\end{abstract}

Key Words - Artificial laryn; Laryngectomy; Fonation

\footnotetext{
1 Trabalho realizado no Hospital das Clínicas da Faculdade de Medicina de Ribeirão Preto - USP

2 Pós-graudando do Departamento de Cirurgia e Anatomia da FMRP-USP

3 Médico residente da Cirurgia de Cabeça e Pescoço do HCRP-FMRP-USP

4 Professor Doutor da disciplina de Cirurgia de Cabeça e Pescoço do Departamento de Oftalmologia, Otorrinolaringologia e Cirurgia de Cabeça e Pescoço do HCRP-FMRP-USP

5 Coordenadora do Cruso de Fonoaudiologia do HCRP-FMRP
} 


\section{INTRODUÇÃO}

Desde a primeira laringectomia total realizada por Billroth $^{1}$, em 1873, a literatura ressalta o interesse de cirurgiões no pesquisar sobre o desenvolvimento da comunicação oral desses pacientes por acreditarem que a falta de comunicação age como uma penalidade psicológica e social. Bailey ${ }^{2,3}$, que em 1976, realizou uma revisão histórica e cronológica da criação e aperfeiçoamento das técnicas cirúrgicas e terapêuticas para tentar a reabilitação da comunicação oral mostrou que ate então as teorias existentes se apresentavam sem resultado funcional. Amatsu, em 1980 e Amatsu e cols., Em $1984^{4,5}$, na tentativa de re-estabelecer a comunicação interpessoal, para estes pacientes, preconizaram a confecção de shunt traqueoesofágico sem uso de prótese externa ou vibrador, que possibilitasse a passagem do ar traqueal para o esôfago na tentativa da capacitar esses pacientes a emitir som esofágico. Este método se mostrou limitado em razão da possibilidade de estenose e da alta incidência de disfagia.

Seguindo os mesmos princípios cirúrgicos de construir uma fístula traqueoesofágica, para a passagem do ar pulmonar para o esôfago, na tentativa de produzir som esofágico, Panje ${ }^{6}$ e Singer \& Blom ${ }^{7}$ idealizaram a prótese de silicone valvulada unidirecional, para ser colocada no interior da fistula. Esta prótese era composta por um dispositivo mecânico destinado a controlar a saída do fluxo aéreo pulmonar para o esôfago, fechando por si a passagem de alimentos para a traquéia e o pulmão.

Singer ${ }^{8}$, em 1992, mudou o formato e confeccionou outra prótese, com os mesmos princípios, ou seja, fazendo a mucosa faringoesofágica vibrar e produzir o som, convertido em fala com a articulação da cavidade oral, lábios, língua e dentes. Este método obteve vasta aceitação, difundindo-se rapidamente.

$\mathrm{O}$ advento da prótese (Figura 1 e 2) diminuta, de material perfeitamente tolerável, valvulada e continente, veio responder a todos os anseios nesta histórica busca de um meio de restabelecer a comunicação das vias aérea e digestiva sem perda da saliva, aspiração e estenose $^{9}$. Hoje é considerada a melhor opção de reabilitação fonatória ${ }^{10}$.

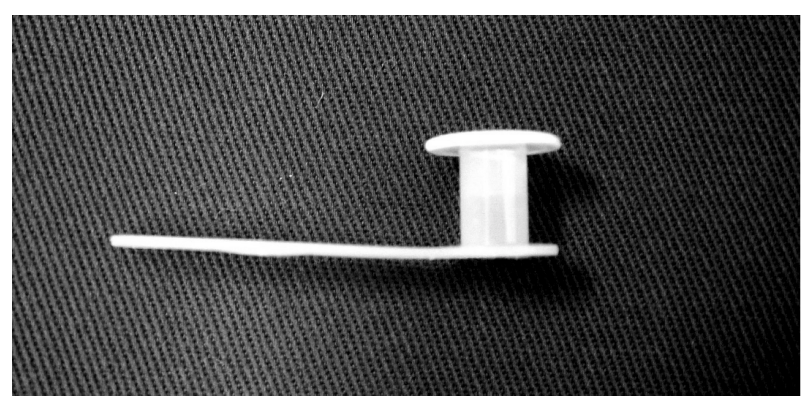

Figura 1 - Prótese Vocal - Vista Lateral.

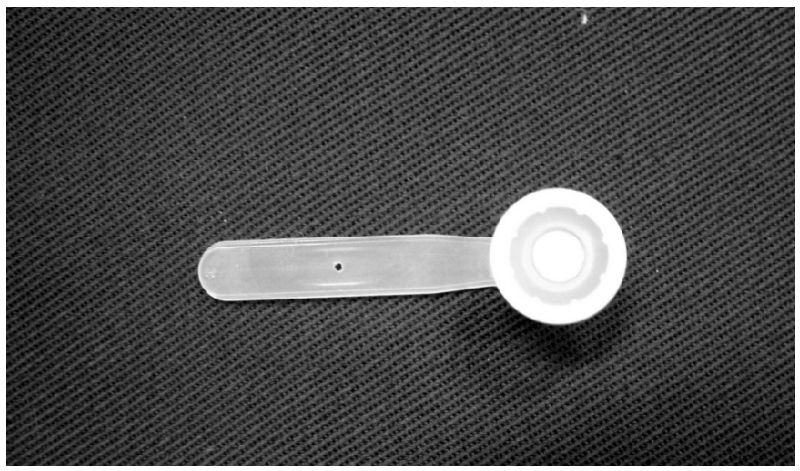

Figura 2 - Prótese Vocal - Vista Posterior.

A decisão pela escolha do uso da prótese está centrada na capacidade anatômica e no desempenho e fisiologia do esôfago e faringe para desenvolver a fonação. Fatores como estenose faríngea, espasmos faríngeos, divertículos, radioterapia prévia, demência, diabetes, doença obstrutiva crônica do pulmão, combinados com os princípios para tratamento oncológico, se somam para chegar-se à melhor decisão.

Entretanto, tem-se observado freqüentes complicações de ordem loco-regional, decorrentes da utilização destas próteses, principalmente em pacientes submetidos a radioterapia ${ }^{11}$. Entre estas complicações estão os vazamentos salivares, seja ao redor da prótese ou pelo interior da mesma, os granulomas e as infecções fúngicas. Embora seja uma conquista para o paciente laringectomizado e os profissionais que, com ele trabalham, alguns aspectos merecem estudo e melhor avaliação.

\section{OBJETIVO}

O presente estudo tem o objetivo de determinar as complicações decorrentes do uso de prótese traqueoesofágica e correlaciona-los com o tipo de prótese e a realização prévia de radioterapia.

\section{MÉTODOS}

Analisou-se pacientes do Serviço de Cirurgia de Cabeça e Pescoço do Hospital das Clínicas da Faculdade de Medicina Ribeirão Preto da Universidade de São Paulo, que foram submetidos a laringectomia total por carcinoma espinocelular e portavam prótese fonatória. Todos os pacientes passaram previamente por terapia fonoaudiológica para aquisição da voz esofágica.

Os integrantes submeteram-se à colocação de prótese traqueoesofágica de silicone valvulada com posicionamento da prótese tipo A (Bloom \& Singer ${ }^{\circledR}$ ) ou tipo B (Provox ${ }^{\circledR}$ ), no período de fevereiro de 2000 a dezembro de 2001. Os pacientes passaram por en- 
trevista com os autores visando obter informações referentes ao tipo de prótese que portavam, e sobre as complicações apresentadas, decorrentes do implante cirúrgico. Todos tinham estudo esofágico previu seja com endoscopia digestiva, seja com seriografia.

A amostra constou de 10 pacientes, todos do sexo masculino, com idade mínima de 55 anos e máxima de 72 anos, com média de 63,5. A prótese tipo A foi utilizada em seis pacientes, enquanto a tipo $\mathrm{B}$, nos quatro remanescentes. Deu-se preferência pela colocação da prótese num segundo tempo, após a cirurgia, em nove dos dez pacientes.

A técnica cirúrgica utilizada para a confecção da fístula traqueoesofágica seguiu-se a descrição de Brown e Rhys-Evans ${ }^{12}$. Consiste na abordagem per-oral, com o auxílio de esofagoscópio rígido. Este progride da faringe ao esôfago, até palpá-lo na parede posterior do traqueostoma. Fazia-se uma punção perfurando a mucosa traqueal e esofágica, com trocarte, número 14 específico, a cerca de $2 \mathrm{~mm}$ da linha de transição cutâneo-mucosa. Após, a mensuração com instrumento próprio, para medir a distância da parede posterior da faringe com a mucosa traqueal e, dessa forma, definir a numeração da prótese a ser posicionada, procedeuse a colocação da mesma.

\section{RESULTADOS}

As complicações observadas em nossos pacientes estão distribuídas na tabela 1 .

Tabela 1 - Tipos de complicações

\begin{tabular}{lcc}
\hline \multicolumn{2}{c}{ TIPOS DE COMPLICAÇÕES } \\
\hline Vazamento & $9(90 \%)$ \\
Infecção & $2(20 \%)$ \\
Dor & $1(10 \%)$ \\
Granuloma traqueal & $1(10 \%)$ \\
Ausência de função & $1(10 \%)$ \\
\hline
\end{tabular}

Cabe acrescentar, entre os casos com vazamento três eram laterais ou peri-prótese e seis eram pelo interior da prótese (Tabela 2).

Tabela 2 - Relação com radioterapia.

\begin{tabular}{ccc}
\hline & RELAÇÃO COM RADIOTERAPIA \\
\hline COMPLICAÇÃo & COM RTX & SEM RTX \\
\hline Vazamento lateral & $1(25 \%)$ & $2(33 \%)$ \\
Vazamento interno & $3(75 \%)$ & $3(50 \%)$ \\
Infecção & ZERO & $2(33 \%)$ \\
Dor & $1(25 \%)$ & ZERO \\
Granuloma traqueal & $1(25 \%)$ & ZERO \\
Ausência de função & ZERO & $1(17 \%)$ \\
\hline
\end{tabular}

O tempo para se realizar a troca das diferentes próteses utilizada está representada na tabela 3 .

Tabela 3 - Relação tempo para primeira troca e tipo de prótese.

\begin{tabular}{|c|c|c|}
\hline \multicolumn{3}{|c|}{ RELAÇÃO TEMPO DE TROCA/TIPO DE PRÓTESE } \\
\hline TEMPO PARA $1^{A}$ TROCA & PRÓTESE A & PRÓTESE B \\
\hline Superior a um ano & Zero & 02 \\
\hline Um ano & 01 & 02 \\
\hline Oito meses & 01 & Não houve \\
\hline Seis meses & 02 & Não houve \\
\hline Quatro meses & 01 & Não houve \\
\hline Dois meses & 01 & Não houve \\
\hline
\end{tabular}




\section{DISCUSSÃO}

Devemos destacar entre as complicações nesta amostra estudada o vazamento de saliva e/ou líquidos da faringe para a traquéia, em permeio à prótese, pois, dos dez pacientes analisados, nove a manifestaram.Estes achados são também referidos em outros trabalhos, como no de Singer ${ }^{8}$ e no de Garth, McRae e Evans ${ }^{11}$.Este vazamento se fez por dentro da prótese em seis casos, sugerindo falha no mecanismo valvular por avaria, por infecção fúngica, ou por outros fatores que prejudiquem a preservação e bom funcionamento da válvula, como por exemplo, a incorreta e/ou inconstante higienização da prótese, com água ou, de preferência, com solução antifúngica.

Em relação ao vazamento por fora da prótese, atribui-se um tamanho inadequado da mesma para o paciente, fazendo com que o mesmo funcione como um pistão ampliando a luz da fístula, permitindo assim o vazamento.Este mecanismo ocorreu em três de nossos pacientes.

Outro motivo para este vazamento está associado à manufatura da prótese, reação alérgica ao material utilizado na sua confecção. Este motivo, no entanto costuma ser exceção não tendo sido detectado em nossos pacientes nenhum caso.

A infecção fúngica ocorreu em dois casos e está diretamente ligada a dois fatores. O primeiro é relativo ao cuidado despendido pelo paciente com a higienização da prótese. Há a necessidade da limpeza continuada da mesma com escova apropriada e utilização de solução antifúngica, o que diminui em muito a contaminação e conseqüente inutilização da prótese. $\mathrm{O}$ segundo, diz respeito a condição clínica do paciente, presença de co-morbidades imunodepressoras como o diabetes, concomitância de tratamentos imunossupressores sejam local ou sistêmico a exemplo de radio e quimioterapia.

A dor crônica registrada em um dos casos pode ocorrer por posicionamento incorreto da prótese, reação inflamatória à sua presença ou ainda infecção local secundária.

Granuloma traqueal foi detectado em um caso e geralmente é devido a reação inflamatória do paciente à prótese, infecção fúngica ou bacteriana e ainda mal posicionamento da prótese.

A ausência de função da prótese se deu em paciente que já fazia uso e muito bem, por muitos anos, da voz esofágica e, provavelmente, não deve ter se sentido estimulado a buscar a adaptação para um outro processo.

Embora seja notório os efeitos loco-regionais da radioterapia (xerostomia, disfagia, irritação da pele, desidratação da mucosa peri-traqueostoma) nossa série não pôde evidenciar diferença estatisticamente significante entre os grupos submetidos à radioterapia e os que não o foram, tanto na incidência das complicações quanto ao tempo de troca.

Ficou evidente a maior durabilidade de uma das marcas empregadas neste estudo tendo sido inclusive a única a registrar tempo de duração entre a colocação e a primeira troca superior a um ano, embora se trate de um trabalho com uma amostra pequena.

Embora haja a recomendação dos fabricantes para a troca da prótese após seis meses, temos pacientes em uso ininterrupto de uma única prótese há mais de dois anos, sem qualquer queixa ou prejuízo fonatório para os mesmos. A realidade sócio-econômica do nosso país e de nossos pacientes faz com que a durabilidade e longevidade da prótese devam ser um fator de extrema importância para a maior difusão e uso da mesma, se considerarmos que o seu preço é um fator limitador para a maioria dos pacientes que poderiam estar se beneficiando de suas vantagens. Portanto, enquanto não se disponibilizar as próteses para a população alvo através do sistema público de saúde, a duração, com qualidade, destas assume importância vital para os pacientes.

A avaliação do trato digestivo alto realizado de rotina em nossos pacientes é importante e pode ser feito pela seriografia ou pela endoscopia digestiva alta. Esta ultima se difundiu nos últimos anos devido a precisão diagnostica e a possibilidade de estudo histopatológico num mesmo exame, o que numa população como a de pacientes tratados de neoplasia de cabeça e pescoço é de grande importância e, já faz parte da rotina de segmento dos pacientes oncológicos da maioria dos serviços seja para detectar e diagnosticar a chance de recidiva, ou um segundo tumor primário.

É objeto de discussão na literatura a forma de colocar a prótese após a cirurgia, pela primeira vez.Gripo, Crespo, Chone e Spina ${ }^{13}$ referem a utilização de esofagoscopia para poder visualizar a punção. Alguns autores optam pela endoscopia digestiva, por sedação, outros por esofagoscopia rígida, com anestesia geral. Esta última permite uma precisão cirúrgica, facilitando o procedimento.Já a colocação por endoscopia digestiva, evita a anestesia geral e é plenamente factível em mãos treinadas.

A prótese pode ser colocada num primeiro tempo ao final do ato operatório quando se faz o shunt traqueoesofágico e se utiliza de uma sonda naso-gástrica número 14 para manter patente este shunt, por em média sete dias, quando então a prótese é aplicada como comentam Gripo, Crespo, Chone e Spina ${ }^{13}$. Em nossos 
pacientes somente um teve a colocação dessa forma, porem a sonda naso-entérica saiu e a fístula se fechou, obrigando-nos a substituir este método pela colocação secundária.

Nossa maior experiência ocorreu com a colocação da prótese em segundo tempo, em média um ano após a cirurgia oncológica. Desta forma acreditamos que de posse do resultado anatomo-patológico e estadiamento patológico pode-se ter melhor avaliação do prognóstico destes pacientes. Nesta época, um ano depois da cirurgia, é tempo suficiente para que já se tenha feito a radioterapia e este paciente esteja em melhores condições clínica e psicológica e, portanto podem se haver com esta nova realidade e ao processo de reconquista da comunicação verbal.

\section{REFERÊNCIAS}

1. Stell P M. The First Laryngectomy. J Laryngol Otol $1975 ; 89 ; 353$.

2. Bailey BJ, Griffiths CM, Everett R. An implanted eletronic laryngeal prothesis. Ann Otol Rhinol Laryngol 1976;85: 472-83.

3. Bailey BJ, Goode RL. New and projected procedures and devices for voice rehabilitation after total laryngectomy. In: Centennial conference on laryngeal cancer, Workshop n. ${ }^{\circ}$ 9. New York, Apleton-Century- Crofts, 1976, p. 576-8.

4. Amatsu M. A one stage surgical technique for postlaryngectomy voice rehabilitation. Laryngoscope 1980;90:1378-86.

05. Amatsu M, Kinish M, Jamir JC. Evalution of speech of laryngectomes after the Amatsu tracheoesophageal shunt operation. Laryngoscope 1984;94:696-701.

06. Panje WR. Prosthetic vocal rehabilitation following laryngectomy: the voice button. Ann Otol Rhinol Laryngol 1981;90:116-20.
07. Singer MI, Blom ED. An Endoscopic Technique for Restoration of Voice After Laryngectomy. Ann Otol Rhinol Laryngol 1980;89:529-33.

08. Singer MI. Voice rehabilitation. In: Cummings CW, Fredrickson JM, Harker LA, Krause CJ, Schuller DE - Otolaryngology Head and Neck Surgery. St. Louis, Mosby Year Book, 1992. V. 2, chapter 118, p. 2190-203.

09. Simpson CB, Postma GN, Stone RE, Ossof RH. Speech outcomes after laryngeal cancer management. Otolaryngol Clin North Am 1997;30:189-205.

10. Delsupehe K, Zink I, Lejaegere M, Delaere P. Prospective Randomized Comparative Study of tracheoesophageal Voice Prostheses: Blom-Singer versus Prostheses Provox. Laryngoscope 1998;108:1561-5.

11. Garth RJN, McRae A, Evans R. Tracheo-esophageal Puncture: A Review of the Problems and Complications. J Laryngol Otol 1991;05:750-4.

12 Brown DH, Rhys-Evans PH. A Simplified method of tracheoesophageal Puncture for Speech Restoration. Laryngoscope 1992;102:579-80.

13. Gripp FM, Crespo NA, Chone CT, Spiro AL. Uso de Prótese para Fonação e Reabilitação do Paciente Laringectomizado. In: Tratado de Cirurgia de Cabeça e Pescoço e Otorrinolaringologia. De Carvalho MB, São Paulo, Editora Atheneu, 2001; capítulo 79, pp 973-978.

\section{Endereço para correspondência}

Leonardo de Souza Kruschewsky

Pós-graudando do Departamento de Cirurgia e Anatomia - FMRP - USP

Campus Universitário - Monte Alegre

14049-990 - Ribeirão preto - SP

e-mail - 